\title{
Frequency and Distribution of Rickettsiae, Borreliae, and Ehrlichiae Detected in Human-Parasitizing Ticks, Texas, USA
}

\section{Elizabeth A. Mitchell, Phillip C. Williamson, Peggy M. Billingsley, Janel P. Seals, Erin E. Ferguson, Michael S. Allen}

To describe the presence and distribution of tickborne bacteria and their vectors in Texas, USA, we screened ticks collected from humans during 2008-2014 for Rickettsia, Borrelia, and Ehrlichia spp. Thirteen tick species were identified, and $23 \%$ of ticks carried bacterial DNA from at least 1 of the 3 genera tested.

$\mathrm{T}$ icks are vectors for a variety of microorganisms, many of which are known agents of zoonotic disease. Although much current research is focused on areas where these diseases are common, it is crucial to collect data from areas with fewer diagnoses of tickborne illness. In Texas, USA, tickborne diseases caused by Rickettsia, Borrelia, and Ehrlichia bacteria are diagnosed less frequently than in some areas of the United States (1); however, those agents have been documented to occur (2), and many medically relevant tick species, capable of carrying and transmitting these pathogens, are established in various geographic areas of Texas (1). Long-term surveillance data encompassing consecutive seasons and a wide geographic range are necessary to ascertain disease transmission risks associated temporally or geographically with established or emerging tickborne pathogens and their vectors. The University of North Texas Health Science Center Tick-Borne Disease Research Laboratory (UNTHSC-TBDL), the primary ticktesting facility for Texas Department of State Health Services Zoonosis Control (TX DSHS), receives ticks continually throughout the year. The data collected from this testing provide an assessment of the prevalence of tick species and associated tickborne bacterial agents collected in Texas.

\section{The Study}

From October 1, 2008, through September 30, 2014, ticks removed from humans were sent by TX DSHS to

Author affiliations: University of North Texas Health Science Center, Fort Worth, Texas, USA (E.A. Mitchell, P.C. Williamson, P.M. Billingsley, J.P. Seals, E.E. Ferguson, M.S. Allen); Creative Testing Solutions, Tempe, Arizona, USA (P.C. Williamson); University of Utah, Salt Lake City, Utah, USA (P.M. Billingsley)

DOI: http://dx.doi.org/10.3201/eid2202.150469
UNTHSC-TBDL, where they were tested by using PCRbased methods, then underwent by DNA sequence analysis to determine the presence of Rickettsia, Borrelia, and Ehrlichia spp. Morphologic identification of tick species was implemented by entomologists at TX DSHS. Ticks that could not be classified morphologically were identified at UNTHSC-TBDL by sequencing mitochondrial $16 \mathrm{~S}$ rDNA (data not shown).

Each tick was sent to UNTHSC-TBDL in an individual collection tube. Upon arrival, ticks were processed according to the laboratory's standard protocol, as described by Williamson et al. (2). After bead pulverization, we extracted DNA using the E.Z.N.A. Mollusc DNA Isolation Kit (Omega Bio-Tek, Norcross, GA, USA) following the manufacturer's protocol.

DNA from each specimen was screened in duplicate by PCR for Rickettsia, Borrelia, and Ehrlichia spp. as previously described (2) by using primers listed in Table 1. PCR products were evaluated, and presumptive-positive amplicons were purified for sequencing (2). Cycle sequencing reactions were performed in both directions by using BigDye Terminator version 3.1 chemistry (Life Technologies, Carlsbad, CA, USA). Dideoxy chain termination products were detected electrophoretically on an ABI 310 or 3130xL Genetic Analyzer (Life Technologies). Sequence analysis was performed by using Sequencher version 4.8/5.0 (GeneCodes, Ann Arbor, MI, USA). Analyzed sequences were compared with reference data in GenBank (http://blast. ncbi.nlm.nih.gov/). Sequences were submitted to GenBank under accession nos. KP861333-KP861347.

The TX DSHS submitted 1,112 ticks to UNTHSCTBDL during October 1, 2008-September 30, 2014, of which 1,062 originated in Texas. Thirteen tick species were identified; most were Amblyomma americanum (55.7\%), followed by Dermacentor variabilis (15.0\%), Rhipicephalus sanguineus (13.0\%), Ixodes scapularis (5.6\%), A. maculatum (5.4\%), and A. cajennense (2.9\%). Approximately $23.3 \%$ of ticks originating in Texas tested positive for DNA from Rickettsia, Borrelia, or Ehrlichia bacteria (Table 2; online Technical Appendix Table, http:// wwwnc.cdc.gov/EID/article/22/2/15-0469-Techapp1. pdf). Of these bacteria, most belonged to spotted fever group rickettsiae (SFGR); A. americanum was the most common tick species found to carry an SFGR agent. The most frequent SFGR sequences detected demonstrated 
Rickettsiae, Borreliae, and Ehrlichiae in Ticks

Table 1. Primers used for screening of human-parasitizing tick specimens, Texas, USA, October 1, 2008-September 30, 2014

\begin{tabular}{|c|c|c|c|c|c|}
\hline Primer name & Gene & Primer sequence, $5^{\prime} \rightarrow 3^{\prime}$ & Specificity & Amplicon, bp & Reference \\
\hline \multicolumn{6}{|l|}{ Borrelia spp. } \\
\hline FlaLL & flaB & ACATATTCAGATGCAGACAGAGGT & Genus & \multirow[t]{2}{*}{664} & (3) \\
\hline FlaRL & flaB & GCAATCATAGCCATTGCAGATTGT & Genus & & (3) \\
\hline FlaLS & flaB & AACAGCTGAAGAGCTTGGAATG & Genus & \multirow[t]{2}{*}{330} & (3) \\
\hline FlaRS & flaB & CTTTGATCACTTATCATTCTAATAGC & Genus & & (3) \\
\hline BL-Fla 522F & flaB & GGTACATATTCAGATGCAGACAGAGGG & B. lonestari & \multirow[t]{2}{*}{660} & (2) \\
\hline BL-Fla 1182R & flaB & GCACTTGATTTGCTTGTGCAATCATAGCC & B. lonestari & & (2) \\
\hline BL-Fla $662 \mathrm{~F}$ & flaB & CTGAAGAGCTTGGAATGCAACCTGC & B. lonestari & \multirow[t]{2}{*}{198} & (2) \\
\hline BL-Fla 860R & flaB & GAGCTAATCCCACCTTGAGCTGG & B. lonestari & & (2) \\
\hline BL-16S 227F & $16 S$ & TCACACTGGAACTGAGATACGGTCC & Genus & \multirow[t]{2}{*}{693} & (2) \\
\hline BL-16S 920R & $16 S$ & GAATTAAACCACATGCTCCACCGC & Genus & & (2) \\
\hline \multicolumn{6}{|l|}{ Rickettsia spp. } \\
\hline Rr.190 70P & rompA & ATGGCGAATATTTCTCCAAAA & Genus & \multirow[t]{2}{*}{532} & (4) \\
\hline Rr.190 602N & rompA & AGTGCAGCATTCGCTCCCCCT & Genus & & (4) \\
\hline BG1-21 & rompB & GGCAATTAATATCGCTGACGG & Genus & \multirow[t]{2}{*}{650} & (5) \\
\hline BG2-20 & rompB & GCATCTGCACTAGCACTTTC & Genus & & (5) \\
\hline \multicolumn{6}{|l|}{ Ehrlichia spp. } \\
\hline Ehr DSB 330F & $d s b$ & GATGATGTCTGAAGATATGAAACAAAT & Genus & \multirow[t]{2}{*}{398} & (6) \\
\hline Ehr DSB 728R & $d s b$ & СTGCTCGTCTATTTTACTTCTTAAAGT & Genus & & (6) \\
\hline Ehr map1F & map1 & ATTTTTACCTGGTGTGTCCTTTTCTGA & Genus & \multirow[t]{4}{*}{873} & (7) \\
\hline Ehr map1R & map1 & ССТТССТССААТТТСТАТАСС & Genus & & (7) \\
\hline Ehr Pmap2F & map1 & GACACCAAGGCAGTATACGG & Genus & & (7) \\
\hline Ehr Pmap2R & map1 & CTAAGTCAGTACCAATACCTGCAC & Genus & & (7) \\
\hline \multicolumn{6}{|l|}{ Tick DNA } \\
\hline $16 \mathrm{~S}-1$ & mt16s & CCGGTCTGAACTCAGATCAAG & Unknown & \multirow[t]{2}{*}{300} & (8) \\
\hline $16 S+2$ & mt16S & TTGGGCAAGAAGACCCTATGAA & Unknown & & (8) \\
\hline
\end{tabular}

$100 \%$ identity to Candidatus Rickettsia amblyommii rompA (GenBank accession no. EF194096). Candidatus $\mathrm{R}$. amblyommii was detected in both $A$. americanum and $A$. cajennense ticks and showed prevalence rates of $30.3 \%$ and $32.3 \%$, respectively. The second most common SFGR rompA sequences were $100 \%$ homologous to the previously termed rickettsial $I$. scapularis endosymbiont, which has been officially named $R$. buchneri (accession no. KP172259) (9). Five A. maculatum specimens contained DNA sequences identical to $R$. parkeri romp $A$ (accession no. KC003476). Sequences that shared 100\% similarity to 1 specific $R$. rhipicephali isolate (accession no. U43803) and $99 \%$ similarity to other $R$. rhipicephali rompA isolates (accession nos. EU109175-EU109178) were obtained from $4 D$. variabilis ticks. Sequences isolated from 2 D. andersoni ticks were identical to $R$. peacockii rompA and rompB (accession nos. FM883671 and
CP001227, respectively). Tick species was confirmed by sequencing mitochondrial 16S rDNA. Sequences from both specimens aligned $99 \%$ with $D$. andersoni (accession no. EU711343) and $94 \%$ with D. variabilis (accession no. L34300). D. andersoni is not known to inhabit Texas $(1,10)$, so this finding could suggest a novel geographic association.

The total prevalence of borreliae detected was $1.1 \%$. DNA sequences sharing $100 \%$ identity to $B$. lonestari were found in 8 A. americanum ticks (1.4\%). As seen by Stromdahl et al., the $B$. lonestari isolates matching sequences in this study depended on the insertion or deletion of a nucleotide triplet, AAG (11). Sequences from 7 tick samples matched $100 \%$ with $B$. lonestari flaB isolates containing the additional triplet (accession no. AY850063), and 1 sequence was identical to $B$. lonestari fla $B$ isolates lacking the triplet (accession no. AY850064). Of the 8 A. americanum

Table 2. Number of positive bacterial DNA sequences identified for each human-parasitizing tick species, Texas, USA, October 1, 2008September $30,2014^{*}$

\begin{tabular}{|c|c|c|c|c|c|c|c|c|c|}
\hline \multirow[b]{3}{*}{ Tick } & \multicolumn{9}{|c|}{ No. positive } \\
\hline & \multicolumn{3}{|c|}{ Borrelia } & \multirow{2}{*}{$\begin{array}{c}\text { Ehrlichia } \\
\text { chaffeensis }\end{array}$} & \multicolumn{5}{|c|}{ Rickettsia } \\
\hline & UNID & burgdorferi & lonestari & & amblyommii† & parkeri & peacockii & rhipicephali & buchneri \\
\hline Amblyomma americanum & 0 & 0 & 8 & 2 & 179 & 0 & 0 & 0 & 0 \\
\hline A. cajennense & 0 & 0 & 0 & 0 & 10 & 0 & 0 & 0 & 0 \\
\hline A. maculatum & 2 & 0 & 0 & 0 & 0 & 5 & 0 & 0 & 0 \\
\hline Dermacentor variabilis & 1 & 0 & 0 & 0 & 0 & 0 & 0 & 4 & 0 \\
\hline D. andersoni & 0 & 0 & 0 & 0 & 0 & 0 & 2 & 0 & 0 \\
\hline Ixodes scapularis & 0 & 1 & 0 & 0 & 0 & 0 & 0 & 0 & 44 \\
\hline Rhipicephalus sanguineus & 0 & 0 & 0 & 0 & 0 & 0 & 0 & 0 & 0 \\
\hline Total & 3 & 1 & 8 & 2 & 189 & 5 & 2 & 4 & 44 \\
\hline
\end{tabular}

${ }^{*}$ Only tick species originating in Texas that tested positive for Borrelia, Ehrlichia, or Rickettsia spp. by DNA sequence analysis are shown. Additionally, 2 A. maculatum ticks from Texas were positive for Panola Mountain Ehrlichia. UNID, unidentified species. †Candidatus species. 
ticks from which the $B$. lonestari sequences were obtained, 6 were co-infected with Candidatus R. amblyommii. DNA extracts from $1 \mathrm{I}$. scapularis tick contained a sequence consistent with $B$. burgdorferi sensu stricto (s.s.) and was co-infected with $R$. buchneri. The $f l a B$ sequence matched $100 \%$ to (accession no. CP002228), and 99\% to (accession no. CP009656) B. burgdorferi s.s. reference sequences. The Borrelia 16S rDNA sequence showed $100 \%$ identity to (accession no. CP009656) and differed by 1 single nucleotide polymorphism from (accession no. CP002228) B. burgdorferi s.s. reference sequences. A $f a B$ gene sequence from 1 D. variabilis tick shared $100 \%$ identity with Candidatus B. texasensis (accession no. AF264901). Samples from 2 A. maculatum ticks showed $f a B$ sequences matching $90 \%$ identity values to $B$. turcica (accession no. AB109243), a reptilian Borrelia sp. Those $f a B$ sequences were identical to a novel Borrelia sp. (accession no. KF395230) previously found in A. maculatum ticks in Mississippi and known to share a phylogenetic clade with B. turcica (12). Borrelia $16 \mathrm{~S}$ rDNA primers produced nonspecific amplification with these 2 samples.

Phylogenetic analysis was performed by using MEGA version 5.1 (http://www.megasoftware.net) using GenBank reference sequences to examine relationships between the Borrelia sp. from this study, B. turcica, and both Lyme disease-associated and relapsing fever borreliae (Figure). The results supported findings by Lee et al. that the novel Borrelia sp. flaB sequences were more closely related to the reptilian Borrelia than the other 2 Borrelia groups (12).

Two A. americanum ticks contained DNA sharing $100 \%$ identity with Ehrlichia chaffeensis $d s b$ (accession no. CP000236). One of these ticks was co-infected with Candidatus R. amblyommii. Prevalence of E. chaffeensis in the A. americanum specimens tested was $0.34 \%$. In addition, 2 of $42 \mathrm{~A}$. maculatum ticks tested for the emerging pathogen
Panola Mountain Ehrlichia sp. (PME) (7) each produced a map 1 sequence that was $100 \%$ homologous to 2 separate PME reference sequences (accession nos. EU272356, EU272358). These sequences differed from each other by 1 single nucleotide polymorphism. This finding represents a novel association, as $A$. americanum is the known vector for PME (7). A subset of $141 \mathrm{~A}$. americanum ticks was also tested for PME, with negative results.

\section{Conclusions}

Frequency of tickborne zoonoses in Texas remains low compared with some regions of the United States. We report the detection of known pathogens along with bacteria of unknown pathogenicity in human-parasitizing ticks commonly found in Texas. Our findings underscore the importance of better characterization and continued surveillance of the frequency and distribution of tick species and the bacterial agents they carry. Continued monitoring in low-risk areas provides data regarding the presence of potential emerging pathogens and vectors not yet commonly identified, which could pose unidentified threats to public health.

\section{Acknowledgments}

We thank Rhonda Roby for advice regarding this work. We also thank Bonny Mayes, Jim Schuermann, Dave Florin, and staff at Texas DSHS Zoonosis Control for collection and taxonomic identification of tick samples.

This project was financially supported by the State of Texas.

Ms. Mitchell is a tick-borne disease analyst at the University of North Texas Health Science Center Tick-Borne Disease Research Laboratory in the Center for Biosafety and Biosecurity. Her current research focuses on detection, identification, and characterization of pathogens and potential emerging agents of human disease.

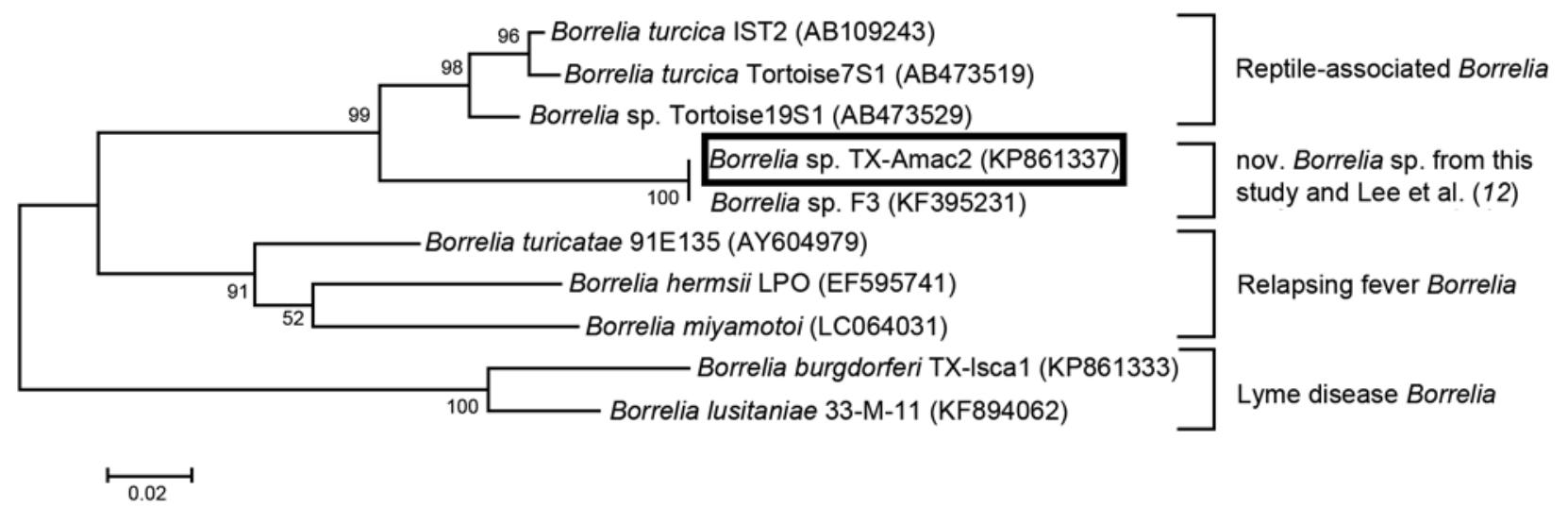

Figure. Maximum-likelihood tree showing that the novel Borrelia sp. identified in Amblyomma maculatum ticks from Texas in this study (box) and from Mississippi (12) shares a closer phylogenetic relationship to $B$. turcica than to to other Borreliae groups. Analysis is based on flaB sequences (267 bp). GenBank accession numbers are shown in parentheses. Tree was constructed using the Tamura 3 -parameter model with a bootstrap value of 1,000 replicates. Scale bar indicates substitutions per nucleotide position. 


\section{References}

1. Centers for Disease Control and Prevention, National Center for Emerging and Zoonotic Infectious Diseases, Division of Vector-Borne Diseases. Geographic distribution of ticks that bite humans [cited 2015 Aug 20]. http://www.cdc.gov/ticks/ geographic_distribution.html

2. Williamson PC, Billingsley PM, Teltow GJ, Seals JP, Turnbough MA, Atkinson SF. Borrelia, Ehrlichia, and Rickettsia spp. in ticks removed from persons, Texas, USA. Emerg Infect Dis. 2010;16:441-6. http://dx.doi.org/10.3201/eid1603.091333

3. Barbour AG, Maupin GO, Teltow GJ, Carter CJ, Piesman J. Identification of an uncultivable Borrelia species in the hard tick Amblyomma americanum: possible agent of a Lyme disease-like illness. J Infect Dis. 1996;173:403-9. http://dx.doi.org/10.1093/ infdis/173.2.403

4. Regnery RL, Spruill CL, Plikaytis BD. Genotypic identification of rickettsiae and estimation of intraspecies sequence divergence for portions of two rickettsial genes. J Bacteriol. 1991;173:1576-89.

5. Eremeeva M, Yu X, Raoult D. Differentiation among spotted fever group rickettsiae species by analysis of restriction fragment length polymorphism of PCR-amplified DNA. J Clin Microbiol. 1994;32:803-10.

6. Doyle CK, Labruna MB, Breitschwerdt EB, Tang YW, Corstvet RE, Hegarty BC, et al. Detection of medically important Ehrlichia by quantitative multicolor TaqMan real-time polymerase chain reaction of the $d s b$ gene. J Mol Diagn. 2005;7:504-10. http://dx.doi.org/10.1016/S1525-1578(10)60581-8

7. Loftis AD, Mixson TR, Stromdahl EY, Yabsley MJ, Garrison LE, Williamson PC, et al. Geographic distribution and genetic diversity of the Erhlichia sp. from Panola Mountain in Amblyomma americanum. BMC Infect Dis. 2008;8:54. http://dx.doi.org/ 10.1186/1471-2334-8-54

8. Black WC IV, Piesman J. Phylogeny of hard- and soft-tick taxa (Acari: Ixodida) based on mitochondrial 16S rDNA sequences. Proc Natl Acad Sci U S A. 1994;91:10034-8. http://dx.doi.org/ 10.1073/pnas.91.21.10034

9. Kurtti TJ, Felsheim RF, Burkhardt NY, Oliver JD, Heu CC, Munderloh UG. Rickettsia buchneri sp. nov., a rickettsial endosymbiont of the blacklegged tick Ixodes scapularis. Int J Syst Evol Microbiol. 2015;65:965-70. http://dx.doi.org/ 10.1099/ijs.0.000047

10. James AM, Freier JE, Keirans JE, Durden LA, Mertins JW, Schlater JL. Distribution, seasonality, and hosts of the Rocky Mountain wood tick in the United States. J Med Entomol. 2006:43:17-24. http://dx.doi.org/10.1093/jmedent/43.1.17

11. Stromdahl EY, Williamson PC, Kollars TM Jr, Evans SR, Barry RK, Vince MA, et al. Evidence of Borrelia lonestari DNA in Amblyomma americanum (Acari: Ixodidae) removed from humans. J Clin Microbiol. 2003;41:5557-62. http://dx.doi.org/10.1128/ JCM.41.12.5557-5562.2003

12. Lee JK, Smith WC, McIntosh C, Ferrari FG, Moore-Henderson B, Varela-Stokes A. Detection of a Borrelia species in questing Gulf Coast ticks, Amblyomma maculatum. Ticks Tick Borne Dis 2014;5:449-52. http://dx.doi.org/10.1016/j.ttbdis.2014.02.004

Address for correspondence: Michael S. Allen, University of North Texas Health Science Center, Department of Molecular and Medical Genetics, 3500 Camp Bowie Blvd, Fort Worth, TX 76107, USA; email: michael.allen@unthsc.edu

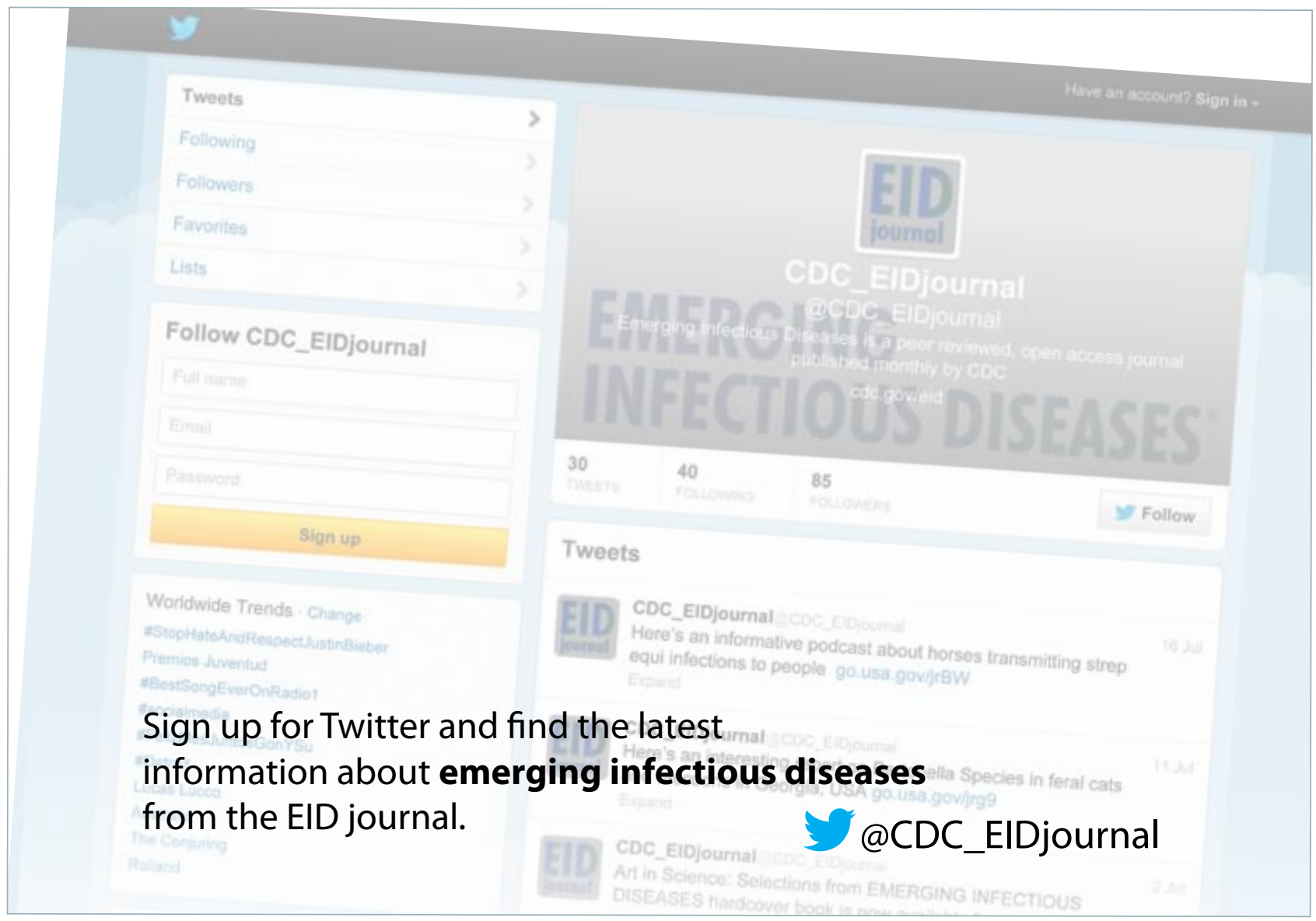

